\title{
Expiation, par Rome, de prodiges survenus dans des cités alliées du nomen Latinum ou des cités alliées italiennes non-latines
}

Les quelques pages qui suivent développent les réflexions qui m’ont été suggérées par l'article de Christopher J. Dart, « The Address of Italian Portents by Rome and the Ager publicus », publié dans le précédent numéro de L'Antiquité Classique'. On ne peut donc les comprendre pleinement qu'après avoir pris connaissance de son travail.

L'objet de nos recherches respectives est différent: Christopher J. Dart s'intéresse à la répartition de l'ager publicus sur le sol italien; j'étudie la prise en charge des prodiges par les autorités romaines ${ }^{2}$. Nous avons cependant été amenés à nous pencher l'un et l'autre sur le dossier de la prise en charge, par Rome, de prodiges survenus dans des cités alliées du nomen Latinum et d'autres cités alliées italiennes ${ }^{3}$.

S'appuyant sur un passage où Tite-Live évoque le refus des autorités romaines, en 169 av. J.-C., de prendre en charge un prodige de la colonie latine de Frégelles au motif qu'il s'était produit in loco peregrino ${ }^{4}$, Christopher J. Dart considère, après $\mathrm{T}$. Mommsen, que seuls les prodiges survenus in agro Romano pouvaient être pris en charge par Rome. Par conséquent, les prodiges des cités alliées latines ou italiennes dont la prise en charge fut acceptée par les autorités romaines se seraient en réalité produits sur des parcelles de l'ager publicus populi Romani et les listes de prodiges qui nous sont parvenues nous permettraient de déterminer la répartition de l'ager publicus sur le sol italien.

Pour ma part, je pense que le refus des autorités romaines de prendre en charge le prodige frégellan de 169 av. J.-C. ne doit pas être compris comme l'application

181 (2012), p. 111-124. J'adresse mes plus vifs remerciements à Christopher J. Dart pour m'avoir fait parvenir son article avant publication; à $\mathrm{M}^{\mathrm{me}}$ Clara Berrendonner, à $\mathrm{M}^{\text {lle }}$ Audrey Bertrand et à mes deux Directeurs de Thèse pour leurs précieux conseils.

2 Dans le cadre d'une Thèse de doctorat intitulée Gouverner par les signes divins: recherches sur l'autorité divinatoire publique, sous la République romaine et au début du Principat, que je prépare sous la direction conjointe de MM. Jean-Michel David (Université Paris 1 Panthéon-Sorbonne) et John Scheid (Collège de France).

3 Par «cités alliées du nomen Latinum », j'entends les quelques cités non annexées du vieux Latium et les colonies de droit latin (A.J. ToYNBEE, Hannibal's Legacy. The hannibalic war's effects on Roman life, Londres/New York/Toronto, 1965, I, p. 249-250, notamment p. 249, n. 3), qui bénéficiaient d'un statut juridique privilégié par rapport aux autres alliés (les quatre privilèges traditionnellement retenus sont le conubium, le commercium, le ius migrandi et, plus tardivement, le ius suffragii : cf. D. KREMER, Ius latinum. Le concept de droit latin sous la République et l'Empire, Paris, 2006, p. 9-45); par " autres cités alliées italiennes » - on comprendra «italiennes » dans un sens géographique -, j'entends toutes les communautés d'alliés italiens non latins.

4 Tite-Live, $A b$ Urbe condita (= LiV.), XLIII, 13, 6. Voir ci-dessous et n. 101. 
d'une prétendue règle juridique préexistante mais comme un avertissement adressé aux Frégellans, diplomatiquement maquillé derrière un prétexte juridique; que la plupart des nombreux prodiges de cités alliées latines et italiennes pris en charge par Rome ne peuvent pas s'être produits in agro publico populi Romani; que ceux, en particulier, qui furent signalés sur les monuments, les portes ou les remparts des cités alliées latines et italiennes survinrent sur des constructions qui ne pouvaient pas relever de la propriété du peuple romain ${ }^{5}$.

Mon étude s'organisera en cinq temps. Je reviendrai d'abord sur les expressions «ager Romanus» et «ager peregrinus», auxquelles Christopher J. Dart a souvent recours, afin de montrer qu'elles risquent, si l'on n'y prend garde, de fausser le débat sur la prise en charge, par Rome, de prodiges étrangers (1). Il me faudra revenir, ensuite, sur le statut de plusieurs des communautés convoquées par Christopher J. Dart pour sa démonstration (2), en particulier sur le statut des cités où des magistrats romains accomplirent des travaux d'urbanisme (3). Je serai alors en mesure d'évaluer l'hypothèse d'une localisation in agro publico populi Romani des prodiges signalés dans les cités alliées latines et italiennes (4). Pour terminer, je replacerai le rejet du prodige frégellan de 169 av. J.-C. dans son contexte socioéconomique et politique, afin d'en dégager quelques pistes d'interprétation (5).

\section{Ager Romanus-ager peregrinus : un binôme historiographique problématique}

Christopher J. Dart écrit, p. 113, « The distinction between ager Romanus and ager peregrinus defined the jurisdictional boundaries between those parts of Italy which were governed by Roman law and those that were under local law ». Cette définition des catégories juridiques ager Romanus et ager peregrinus renvoie à un usage davantage historiographique qu'historique. Elle est, en outre, discutable : elle passe sous silence à la fois l'autonomie interne - certes limitée - des municipes, qui conservaient, en dépit de la perte de leur souveraineté, leurs organes d'administration locale, et, à l'inverse, la place prise par le droit romain dans la législation des colonies latines, pourtant formellement indépendantes ${ }^{6}$. Il serait plus exact d'entendre l'ager Romanus, compris dans son acception historiographique, comme l'ensemble des territoires des communautés ayant reçu la citoyenneté romaine, qu'il s'agisse de la civitas sine suffragio ou de la civitas optimo iure. À l'inverse, l'ager peregrinus correspondrait aux territoires des communautés restées hors de la citoyenneté romaine. Mais l'on constate alors que l'usage historiographique que l'on fait habituellement de ces deux expressions «territorialise » artificiellement un statut juridique censé s'appliquer en fait aux personnes, glissement contre lequel $\mathrm{C}$. Nicolet nous mettait déjà en

5 J'ai déjà abordé ces problèmes dans « Expiation, par les autorités romaines, de prodiges survenus en terre étrangère. Quelques réflexions sur le statut juridique des territoires et des communautés alliés, et sur le processus de "romanisation" ", Paris, 2009, p. 168-178, en ligne : URL : www.cairn.info/revue-hypotheses-2009-1-page-169.htm. (état : décembre 2012).

6 Pour les municipes, voir M. HUMBERT, Municipium et civitas sine suffragio. L'organisation de la conquête jusqu'à la Guerre Sociale, Rome, 1978 (Collection de l'École Française de Rome, 36), p. 287-333, particulièrement p. 304-309. Pour les colonies latines, voir ci-dessous et D. KREMER, o.c. (n. 3), p. 91-95. 
garde $^{7}$. C'est pourquoi il conviendrait peut-être d'abandonner l'usage historiographique du binôme ager Romanus-ager peregrinus pour ne pas risquer de fausser notre lecture des sources.

Les expressions ager Romanus et ager peregrinus y ont en effet un sens très différent. Dans leur acception technique, elles relèvent du droit augural. Un célèbre passage de Varron nous livre, à cet égard, de précieuses définitions : "Comme l'exposent nos augures publics, il y a cinq types de territoires : le « romain » (Romanus), le « gabin» (Gabinus), l' « étranger» (peregrinus), l' « ennemi » (hosticus) et l'« incertain » (incertus). Le « romain » (Romanus) tire son nom de Romulus, d'où « Rome»; le «gabin» (Gabinus), de l'oppidum de Gabies ; l' « étranger» (peregrinus) est le territoire pacifié qui est à part du « romain » et du « gabin » parce que, dans ces derniers, on observe les auspices d'une seule et même manière; il fut nommé peregrinus à partir de pergere [aller en avant], c'est-à-dire «s'avancer »: c'était dans sa direction, en effet, qu'ils faisaient leurs premières avancées ${ }^{8}$ hors du territoire romain ; en conséquence, le « gabin » aussi est « étranger », mais parce qu'il a des auspices propres il est distingué du reste ; l'« ennemi » (hosticus) tire son nom des « ennemis » (hostes) ; l' « incertain » (incertus) est celui pour lequel on ignore duquel de ces quatre [types de territoires] il relève ${ }^{9} »$.

Comme on le voit dans cette définition, le terme ager s'applique à des territoires très limités, conformément au modèle territorial de la cité antique, associant étroitement un pôle urbain à son territoire rural. Tite-Live se conforme à cet usage quand il parle d'ager Veiens ${ }^{10}$, Amiternus $^{11}$, $^{1}$ apenas $^{12}{ }^{12}$ Crustuminus $^{13}{ }^{13}$ Campanus $^{14}$,

7 C. Nicolet, Rome et la conquête du monde méditerranéen, Paris, $1995^{8}$ [1979] (Nouvelle Clio), I. «Les structures de l'Italie romaine», p. 275 : « il ne faut jamais oublier qu'il s'agit de statuts personnels, non de territoires. »

8 Ma traduction cherche à rendre l'idée d'une expansion territoriale, induite par la définition de l'ager peregrinus comme «ager pacatus » (territoire pacifié) et à ne pas réduire progredior à l'idée d'un simple déplacement spatial. Cf. O. SACCHI, L'ager Campanus antiquus. Fattori di trasformazione e profili di storia giuridica del territorio dalla 'Mesogeia' alla 'centuriatio' romana, Naples, 2004 (Pubblicazioni della Facoltà di Giurisprudenza della seconda università di Napoli, 25), p. 155 et n. 14.

9 VARron, De Lingua latina, 5, 33 : Ut nostri augures publici disserunt, agrorum sunt genera quinque: Romanus, Gabinus, peregrinus, hosticus, incertus. Romanus dictus unde Roma ab Rom $<u l>o$; Gabinus ab oppido Gabi $<i>s$; peregrinus ager pacatus, qui extra Romanum et Gabinum, quod uno modo in his serv<a $>$ ntur auspicia; dictus peregrinus a pergendo, id est a progrediendo : eo [quod] enim ex agro Romano primum progrediebantur. Quocirca Gabinus quoque peregrinus, sed quod auspicia habe[n]t singularia, ab reliquo discretus; hosticus dictus ab hostibus; incertus is, qui de his quattuor qui sit ignoratur. Trad. pers. Cf. P. CATAlAnO, "Aspetti spaziali del sistema giuridico-religioso romano", $A N R W$ II.16.1 (1978), p. 492-498 ; O. SACCHI, o.c. (n. 8), p. 149-158.

10 LiV., XLI, 21, 12 ; XLIV, 18, 6.

11 LIV., XXI, 62, 5.

12 LiV., XXVII, 4, 14-15.

13 LiV., XLI, 9, 5.

14 LIV., XLI, 21, 13. 
Veliternus $^{15}$, etc., à une époque où les territoires concernés ont été annexés par Rome depuis longtemps ${ }^{16}$. Il n'emploie pas autrement l'expression ager Romanus : Bis in exitu anni eius lapidatum esse nuntiatum est, <semel $>$ in Romano agro, semel in Veient $i^{17}$. Jamais, à ma connaissance, ager Romanus ne désigne, dans les sources républicaines, l'ensemble des territoires annexés par Rome ${ }^{18}$. Pour ces derniers, en effet, comme l'avait déjà noté en son temps T. Mommsen, il n'existe pas d'expression exacte $^{19}$. Et c'est sans doute ce vide terminologique qui suscita la création historiographique du binôme ager Romanus-ager peregrinus. Je renoncerai, pour ma part, à y recourir, jugeant moins risqué et plus pertinent d'opposer les cités bénéficiant de la citoyenneté romaine, optimo iure ou sine suffragio, à celles qui, relevant du droit latin ou d'un droit pérégrin, en étaient exclues.

\section{Quelques précisions sur le statut de certaines communautés}

Il convient maintenant de revenir sur le statut juridique que Christopher J. Dart attribue à certaines des communautés qu'il convoque pour son raisonnement. Plusieurs de ces attributions sont erronées, parfois au détriment de sa démonstration.

\subsection{Capoue, « a legal limbo » entre 211 et 189 ?}

Il paraît exagéré de qualifier le statut de Capoue, entre 211 et 189 av. J.-C., de « vide juridique », comme le fait Christopher J. Dart, p. 119, en parlant de « legal limbo ». On sait en effet qu'en 211, le Sénat décida la confiscation du territoire de Capoue $^{20}$, la suppression de ses institutions politiques et de son autonomie munici-

15 LiV., XXX, 38, 8.

16 Cf. A. ALFÖLDI, Early Rome and the Latins, Ann Arbor, 1965, p. 304-305.

17 LIV., XLIV, 18, 6. Cf. Liv., XLI, 21, 11-12.

18 Liv., XXVII, 5, 15 (Illa disceptatio tenebat quod consul in Sicilia se M. Valerium Messallam qui tum classi praeesset dictatorem dicturum esse aiebat, patres extra Romanum agrum - eum autem Italia terminari - negabant dictatorem dici posse) n'implique pas que l'ager Romanus s'étendait alors à toute l'Italie, mais qu'il n'était pas possible de créer, par fiction juridique, une parcelle d'ager Romanus en dehors de l'Italie. En effet, si toute l'Italie, en 210 av. J.-C., avait relevé de l'ager Romanus, on ne comprendrait pas la nécessité, pour le consul Crispinus, de recourir deux ans plus tard, en Campanie, à la fiction juridique de l'ager Romanus (LiV., XXVII, 29, 5). Cf. T. MomMSEN, Le Droit public romain, trad. fr., Paris, 1893, III, p. 173 et n. 2 ; P. CATAlano, l.c. (n. 9), p. 529 ; O. SACCHI, o.c. (n. 8), p. 162 et n. 28 ; p. $167-168$ et n. 42 .

19 T. MommSen, o.c. (n. 18), 1889, $\mathrm{VI}^{2}$, p. 476-479. Cf. P. CATAlano, l.c. (n. 9), p. 493 ; O. SACCHI, o.c. (n. 8), p. 152.

20 Pour une discussion approfondie du statut juridique conféré à l'ager Campanus par le sénatus-consulte de 211 av. J.-C., voir A. LinTotT, Judicial reform and land reform in the Roman Republic. A new edition, with translation and commentary, of the laws from Urbino, Cambridge, 1992, p. 203 et surtout O. SACCHI, o.c. (n. 8), p. 191-234, avec la bibliographie antérieure, qui considère qu'il fut seulement publicatus, c'est-à-dire "confisqué », et non pas rendu publicus à proprement parler, catégorie juridique qui ne lui paraît pas être en place avant les Gracques (p. 171-189 et p. 214). Sur l'utilisation anachronique de cette catégorie dans la tradition annalistique et sur le caractère très progressif de son élaboration, voir aussi 
pale, et sa réduction à l'état de préfecture, c'est-à-dire d'une simple circonscription judiciaire dépendant d'un préfet iure dicundo envoyé chaque année de Rome ${ }^{21}$. Quintus Fulvius Flaccus fut chargé de vendre les biens des principes capouans et d'affermer les terres confisquées ${ }^{22}$, affermage qui fut poursuivi par les censeurs de $209^{23}$. En 205, les questeurs reçurent l'ordre de « vendre $»^{24}$ la partie de l'ager Campanus située entre la fossa Graeca et la mer ${ }^{25}$. En 199, ce fut au tour des censeurs de « vendre » le territoire capouan qui s'étendait au pied du Mont Tifata. Ils déduisirent peut-être, en outre, une colonie de 300 colons à Castrum Portorium ${ }^{26}$. En 197-194, un triumvirat coloniis deducendis fut chargé de fonder cinq colonies de 300 colons sur la côte maritime, Pouzzoles, Vulturne, Literne, Salerne et Buxentum ${ }^{27}$. En 189 av. J.-C., après une suspension provisoire de leur civitas, les Capouans, comme les autres Campaniens qui avaient fait défection en 216 av. J.-C., obtinrent d'être de nouveau inscrits, comme citoyens, sur les listes du cens, mais à Rome même ${ }^{28}$.

Un tel statut, loin d'être un vide juridique, n'empêche certes pas d'interpréter les nombreux signalements de prodiges rapportés entre 211 et 189 av. J.-C. comme le signe d'une volonté des Capouans de marquer leur fidélité à Rome ${ }^{29}$. En revanche, dans la mesure où Capoue n'avait plus, et depuis longtemps, le statut d'alliée, son cas ne saurait être utilisé pour prouver que les prodiges alliés pris en charge par Rome se produisirent en fait in agro publico.

E. Hermon, Habiter et partager les terres avant les Gracques, Rome, 2001 (Collection de l'École Française de Rome, 286), en particulier p. 141-199 et 289, qui considère, pour sa part, que le concept était établi dès la fin du IV siècle av. J.-C. La transformation de la Sabine en ager publicus, à la fin de la troisième guerre samnite, en aurait constitué l'une des premières applications historiques.

21 CicÉron (= Cic.), De Lege agraria, I, 19 ; II, 88 ; LiV., XXVI, 16, 7-10 ; Velleius Paterculus, Histoire Romaine (= Vell. Pat.), II, 44, 4 ; Festus, De Verborum significatu (=FEST.), s.v. Praefecturae, p. 262 L. Pour cette « réduction » de Capoue au statut de simple "préfecture », voir M. HUMBERT, o.c. (n. 6), p. 355-402, qui a bien montré, notamment p. 366367 et n. 30, que le statut de préfecture n'était pas incompatible avec celui de municipe : seules Capoue et ses alliées, en raison de leur défection, furent réduites à l'état de préfectures, perdant leurs institutions politiques municipales pour ne conserver que le statut de préfectures judiciaires.

22 LiV., XXVI, 34, 11 et XXVII, 3, 1.

23 LIV., XXVII, 11, 8.

24 Sur le sens technique des «ventes» de portions d'ager Campanus en 205 et 199 av. J.-C., voir FeST., s.v. Ven<ditiones>, p. 516 L et O. SACCHI, o.c. (n. 8), p. 210-211.

${ }_{25}$ LIV., XXVIII, 46, 4-5.

26 Liv., XXXII, 7, 3.

27 Liv., XXXIV, 45, 1-2 ; Vell. PAT., I, 15, 3. Cf., pour la fondation de Pouzzoles, CIL X, $1781=C I L \mathrm{I}^{2}, 698=I L S, 5317$

28 LiV., XXVI, 34, 7 et XXXVIII, 28, 4. Cf. M. HuMBert, o.c. (n. 6), p. 312, n. 96.

29 C.J. DART, p. 119. 


\subsection{Atella et Calatia}

Atella n'était pas une colonie romaine, comme l'écrit Christopher J. Dart, p. 121: «Both communities [Minturnae and Atella] were citizen colonies », et Calatia ne devint pas une communauté romaine seulement en 59 av. J.-C., comme il l'affirme, p. 117 : «Calatia did not become a Roman citizen community until 59 $\mathrm{BCE}$ ».

Ces deux cités campaniennes, en effet, devinrent très probablement des municipia sine suffragio, au même titre que Capoue, Suessula et Cumes, dans les années qui suivirent la défaite des Campaniens face aux Romains en 340 av. J.-C. ${ }^{30}$

En 211 av. J.-C., en punition de leur défection en faveur d'Hannibal, elles perdirent très vraisemblablement, comme Capoue, leurs institutions politiques et leur autonomie municipale pour être réduites au statut de préfectures, c'est-à-dire de simples circonscriptions judiciaires ${ }^{31}$. La présence d'Atella et de Calatia sur la liste des préfectures de Festus, si elle ne suffit pas à prouver qu'elles perdirent alors leur statut municipal, constitue toutefois un premier indice ${ }^{32}$, que confirment les similitudes entre leur sort et celui de Capoue : comme elle, elles firent défection après la bataille de Cannes ${ }^{33}$, puis capitulèrent à sa suite ${ }^{34}$ et subirent, en la personne de leurs magistrats et sénateurs, les mêmes châtiments ${ }^{35}$; la déportation des Atellani abonde dans le même sens ${ }^{36}$.

Par la suite, en 59 av. J.-C., César déduisit une colonie de vétérans à Calatia ${ }^{37}$. Atella, quant à elle, n'accéda jamais, avant les derniers temps de la République en tout cas, au statut de colonie romaine : Cicéron, en 54 et en 46-45 av. J.-C., parle d'Atella comme d'un simple municipium ${ }^{38}$. L'allusion des Libri coloniarum, I, X, 5 ${ }^{39}$, par

30 Cf. LiV., VIII, 14, 10-11 ; Vell. PAT., I, 14, 3, où les Calatini et les Atellani sont sans doute compris parmi les Campani recevant le droit de civitas sine suffragio. Cf. J. BELOCH, Campanien. Topographie, Geschichte und Leben der Umgebung Neapels im Alterthum, Berlin, 1879, p. 370 et 379 ; J. HEURGON, Recherches sur l'histoire, la religion et la civilisation de Capoue préromaine des origines à la deuxième guerre punique, Paris, 1942 (BEFAR, 154), p. 189-190 ; A.J. ToYnBEe, o.c. (n. 3), I, p. 139, n. 5 et 6 ; M. HuMBERT, o.c. (n. 6), p. 205 et n. 192 ; O. SACCHI, o.c. (n. 8), p. 22. Selon que l'on suit la datation de Tite-Live ou celle de Velleius Paterculus, on datera respectivement l'octroi de la civitas sine suffragio de 338 av. J.-C. ou de 334 av. J.-C. : cf. M. HuMBERT, o.c. (n. 6), p. 173, n. 62 et p. 195, n. 146 ; A.J. TOYNBEE, o.c. (n. 3), I, p. 139, n. 6.

31 Cf. J. Beloch, o.c. (n. 30), p. 371 et 379-380 ; J. Heurgon, o.c. (n. 30), p. 239, n. 4 ; A.J. ToynBeE, o.c. (n. 3), I, p. 242 et 410 ; II, p. 121-128; M. HuMBERT, o.c. (n. 6), p. 366-372. Sur cette « réduction » à l'état de simple préfecture judiciaire, voir n. 21 .

32 FEST., s.v. Praefecturae, p. 262 L.

33 LiV., XXII, 61, 11 et Silius Italicus, Punica, XI, 14.

34 LiV., XXVI, 16, 5 ; POLYBE, Histoires, IX, 9, 10 a.

35 LIV., XXVI, 34, 11.

36 LiV., XXVII, 3, 7 ; ApPIEN (= App.), Le Livre d'Annibal, 49, 211.

37 Cic., Epistulae ad Atticum, XVI, 8, 1 = 418 Shackleton Bailey ; Vell. PAT., II, 61, 2 ; NiCOL. DAM., 31, 136 ; APP., Guerres civiles, III, 40, 164-165.

38 Cic., Epistulae ad fratrem Quintum, II, 13/12, $3=17$ Shackleton Bailey (54 av. J.-C.) ; Epistulae ad familiares, XIII, 7, $1=320$ Shackleton Bailey (46-45 av. J.-C.). 
ailleurs problématique ${ }^{40}$, à la déduction d'une colonie augustéenne à Atella, ne fait pas pour autant d'Atella une colonie sous la République. Quant à l'affirmation de Christopher J. Dart, p. 121 et n. 62, selon laquelle Atella fut habitée par des citoyens romains à partir de 210 av. J.-C. (« the residents of Atella had been citizens since $210 »)$, date de la déportation des Atellani à Calatia et de l'installation des habitants de Nuceria à leur place, non seulement elle n'autorise pas à faire d'Atella une colonie romaine à partir de 210 , comme il semble le sous-entendre, mais elle mérite surtout d'être discutée, les Nucerini n'étant à cette date que des alliés de Rome et non des citoyens romains. Il n'est toutefois pas impossible d'imaginer, dans l'hypothèse d'une implantation durable des Nucerini à Atella, qu'ils reçurent par la suite la citoyenneté romaine $^{41}$.

Si l'erreur relative au statut d'Atella n'a pas de conséquence sur le raisonnement de Christopher J. Dart, il en va tout autrement de l'erreur relative au statut de Calatia, dont le cas, tout comme celui de Capoue, ne saurait être utilisé pour prouver que les prodiges alliés pris en charge par Rome se produisirent en fait in agro publico. J'y reviendrai.

\subsection{Anagni}

Je signale une dernière erreur factuelle: Anagnia ne devint pas un municipe uniquement en 57 av. J.-C., comme l'écrit Christopher J. Dart, p. 121, « As Anagnia did not become a municipium until $57 \mathrm{BCE}$ », en s'appuyant, à la note 59, sur Cicéron, De Domo sua, 81, qui ne dit rien de tel : «Mais toi, ravisseur des droits civiques, tu as même porté une loi sur les délits criminels, pour plaire à je ne sais quel Menulla d'Anagni, qui, en reconnaissance, t'a élevé une statue dans ma maison, sans doute pour que ce lieu même, témoin d'une telle iniquité, démentît la loi et l'inscription de la statue ; cette affaire a causé aux habitants les plus distingués du municipe beaucoup plus d'affliction que les crimes commis à Anagni par ce même gladiateur. $^{42}$ " Anagnia ayant reçu la civitas sine suffragio en 306 av. J.-C. ${ }^{43}$, c'est dès cette date qu'il convient d'en faire un municipe romain ${ }^{44}$.

39 La numérotation I, X, 5 = p. 230 Lachmann, est empruntée à l'édition des Libri coloniarum de C. Brunet, D. Conso, A. Gonzales, T. Guard, J.-Y. Guillaumin et C. Sensal, Besançon, 2008.

40 Cf. C. HÜlsen, s.v. Atella, RE II.2 (1895), col. 1914 ; P.A. BRUnT, Italian Manpower, 225 B.C.-A.D. 14, Oxford, 1971, p. 337 et n. 1.

41 H. PHILIPP, s.v. Nuceria 1), RE XVII.1 (1936), col. 1236 ; C. HÜLSEN, s.v. Atella, RE II.2 (1895), col. 1914 ; A. J. TOYNBEE, o.c. (n. 3), I, p. 410, n. 4 ; II, p. 550, n. 4.

${ }^{42}$ At tu etiam, ereptor civitatis, legem de iniuriis publicis tulisti, Anagnino nesciocui Menullae pergrat[i]am, qui tibi ob eam legem statuam in meis aedibus posuit, ut locus ipse in tanta tua iniuria legem et inscriptionem statuae refelleret, quae res municipibus [Anagninis] ornatissimis multo maiori dolori fuit quam quae idem ille gladiator scelera Anagniae fecerat. Trad. P. Wuilleumier, Paris, 1952 (Collection des Universités de France = CUF).

43 LIV., IX, 43, 24.

44 M. HUMBERT, o.c. (n. 6), p. 37-39 et 213-216. 


\section{Des ouvrages urbains de magistrats romains dans les cités alliées?}

Christopher J. Dart écrit, p. 112, « the observation that many temples, walls, and other parts of many Latin and allied Italian cities would have to have been public property is not a valid objection, as public infrastructure such as roads, temples and fortifications built with Roman state funds would have indeed been Roman property. » et, p. 118, «First, all walls, gates and temples paid for with public funds within Roman colonies and municipia were Roman public property. Second, all roads, highways and other building works commissioned by the Roman censors or paid for by the Roman state were public property, regardless of their actual location ».

Personne ne contestera que les ouvrages engagés, sur des fonds publics, par les censeurs romains ou, en l'absence de censeurs, par d'autres magistrats, en priorité les consuls ou les préteurs $^{45}$, fussent propriété du peuple romain. Il n'est pas moins évident que la construction de routes à travers l'Italie impliquait de les faire passer sur les territoires des alliés latins et italiens, sans doute avec leur accord. Il est, en revanche, plus problématique d'affirmer que les censeurs ou d'autres magistrats de Rome pouvaient intervenir dans la construction des murs, des portes et des bâtiments mêmes des cités alliées du nomen Latinum ou des cités alliées italiennes. Non seulement cela va à l'encontre de l'indépendance qui leur était reconnue par Rome, mais surtout nous n'en avons, à ma connaissance, aucune attestation. Des huit cités pour lesquelles des travaux censoriaux hors de Rome sont attestés, aucune n'a le statut d'alliée, mais toutes sont de droit romain ${ }^{46}$. Ainsi, en 184 av. J.-C., le censeur Lucius Valerius Flaccus fit construire une route qui longeait les collines de Formies ${ }^{47}$, municipe romain ayant reçu quatre ans plus tôt, en 188 av. J.-C., la citoyenneté optimo iure $^{48}$. En 179 av. J.-C., le censeur Marcus Aemilius Lepidus fit bâtir une digue près de Tarracine ${ }^{49}$, colonie romaine depuis 329 av. J.-C ${ }^{50}$. En 174 av. J.-C., les censeurs Quintus Fulvius Flaccus et Aulus Postumius Albinus mirent en adjudication la construction des murs de Calatia et d'Auximum ${ }^{51}$ : Calatia, déchue de son statut municipal sine suffragio depuis 211 av. J.-C., comme on l'a vu, n'était plus qu'une préfecture judiciaire, dont le territoire avait été confisqué par Rome ; Auximum, en dépit d'une indication peu fiable de Velleius Paterculus, était vraisemblablement déjà une colonie romaine à cette date ${ }^{52}$. La même année, Quintus Fulvius Flaccus fit

45 T. Mommsen, o.c. (n. 18), 1894, IV, p. 110-111; cf. p. 158-159. J. SuOlahti, The Roman censors. A study on social structure, Helsinki, 1963 (Annales Acad. Scient. Fennicae, série B, vol. 117), p. 70 et n. 3. À la fin de la République, la construction des voies romaines passa sous la responsabilité des gouverneurs de provinces: T. MOMMSEN, o.c. (n. 18), 1894, IV, p. 143-144. J. SuOlahti, o.c. (ci-dessus), p. 65 et n. 5.

46 Déjà, T. MOMMSEN, o.c. (n. 18), 1894, IV, p. 114, n. 1.

47 Liv., XXXIX, 44, 6.

48 Liv., XXXVIII, 36, 7.

49 LiV., XL, 51, 2.

50 Liv., VIII, 21, 11 (Tarracina est l'antique Anxur) ; Vell. PAT., I, 14, 4.

51 LiV., XLI, 27, 10.

52 La date de la déduction de la colonie romaine d'Auximum indiquée par Velleius Paterculus, I, 15, 3 (157 av. J.-C.) est fort peu vraisemblable, ne serait-ce qu'en raison des deux mentions qu'en fait Tite-Live à une date antérieure, en 175 av. J.-C. pour un prodige (LiV., 
construire un temple de Jupiter et une rue pavée à Pisaurum ${ }^{53}$, colonie romaine fondée dix ans plus tôt, en 184 av. J.-C. ${ }^{54}$; un temple de Jupiter à $F u n d i^{55}$, municipe romain ayant reçu quatorze ans plus tôt, en 188 av. J.-C., la citoyenneté optimo iure ${ }^{56}$; un aqueduc à Potentia ${ }^{57}$, colonie romaine fondée dix ans plus tôt, en 184 av. J.-C. ${ }^{58}$; des habitations à Sinuessa [?] $]^{59}$, colonie romaine fondée en 296 ou 295 av. J.-C. ${ }^{60}$. Quant aux travaux consulaires attestés en Lucanie, près de la voie Popilia-Annia, par une inscription du troisième quart du $\mathrm{II}^{\mathrm{e}}$ siècle av. J.-C. retrouvée à Polla (Forum Popilii), ils furent effectués in agro publico, sur une des très nombreuses terres confisquées aux Lucaniens après la deuxième guerre punique ${ }^{61}:$ «J'ai fondé ce Forum et construit des bâtiments publics ${ }^{62} »$. Il n'est donc pas possible de s'appuyer sur de prétendus travaux de censeurs ou d'autres magistrats romains dans les cités alliées latines ou italiennes pour dire que les remparts et les temples situés sur leur territoire étaient la propriété du peuple romain.

\section{Prodiges survenus in agro publico ou en terre alliée ?}

Alors que Christopher J. Dart souhaite prouver, après T. Mommsen ${ }^{63}$, que les prodiges signalés dans des cités alliées du nomen Latinum ou des cités alliées italiennes non-latines se déroulèrent en fait sur des terres relevant, d'un point de vue juridique, de l'ager publicus, c'est-à-dire de la propriété du peuple romain, il ne cite, à l'exception de Frégelles, que des cités déjà rattachées à la civitas romana lorsque leurs prodiges furent pris en charge par Rome : Calatia (p. 117), Amiternum (p. 118),

XLI, 21, 12) et en 174 pour les travaux censoriaux qui nous occupent. Cf. U. LAFFI, « Linee di sviluppo della colonizzazione romana dalla fine della guerra annibalica all'età graccana ", in Iuris Vincula. Studi in onore di Mario Talamanca, Naples, 2001, IV, p. 524 [repris dans Colonie e municipi nello stato romano, Rome, 2007, p. 41]. Auximum connut peut-être une phase pré-coloniale, mais nous n'en savons rien (A.J. TOYNBEE, o.c. (n. 3), II, p. 208 et n. 2). Même à supposer que ce fût le cas, il y a peu de chances pour que la cité ait échappé à l'annexion romaine qui suivit la révolte picentine de 269 av. J.-C. : seules Asculum et, peutêtre, Ancône purent y échapper et conserver leur statut d'alliées. Cf. A.J. ToYNBEE, o.c. (n. 3), I, p. 154, n. 5. Pour Ancône, voir U. Moscatelli, "Ancona », Picus 3 (1983), p. 272 et S. Sebastiani, « Sulle fasi urbane di Ancona antica », Archeologia Classica 35 (1983), p. 289.

53 LIV., XLI, 27, 11.

54 Liv., XXXIX, 44, 10 ; Vell. PAT., I, 15, 2.

55 LIV., XLI, 27, 11.

56 LIV., XXXVIII, 36, 7.

57 LiV., XLI, 27, 11

58 Liv., XXXIX, 44, 10 ; Vell. PAT., I, 15, 2.

59 LIV., XLI, 27, 12. Le texte des manuscrits est très corrompu à cet endroit : il y est aussi question de la construction d'égouts, d'un mur d'enceinte, de portiques, de boutiques, etc.

60 Liv., X, 21, 8 (296 av. J.-C.) ; Vell. PAT., I, 14, 6 (295 av. J.-C.).

61 A.J. TOYNBEE, o.c. (n. 3), II, p. 117-121, en particulier p. 119-120.

$62 C I L \mathrm{I}^{2}, 638=C I L \mathrm{X}, 6950=I L S, 23:$ Forum aedisque poplicas heic fecei.

63 T. Mommsen, "Epistula de Romanorum prodigiis ad Ottonem Jahnium », in id., Gesammelte Schriften, Berlin, 1909, VII, p. 168-174. Cf. E. RUOFF-VÄÄNÄNEN, « The Roman Public Prodigia and the Ager Romanus », Arctos 7 (1972), p. 139-162. 
Gabies (p. 118), Capoue (p. 119), Lanuvium (p. 120), Anagnia (p. 121), Minturnae (p. 121), Atella (p. 121), Véies (p. 121), Caere (p. 121). Les cités alliées latines ou italiennes ayant rapporté des prodiges à Rome ne manquent pourtant pas: Veit Rosenberger évalue la proportion de ces prodiges à environ $21 \%$ des prodiges expiés par Rome ${ }^{64}$. Christopher J. Dart en a bien conscience puisqu'il dit lui-même, p. 112 : « Many modern scholars have rightly observed that numerous portents recorded by Livy were reported in reference to communities of Latin or allied status ». Dans ces conditions, on est en droit de s'étonner qu'aucune de ces cités ne soit convoquée pour appuyer sa démonstration.

L'explication est simple : le seul cas abondant dans son sens est le prodige lucanien de 200 av. J.-C., localisé in agro publico ${ }^{65}$. Les alliés lucaniens, qui étaient massivement passés dans le camp d'Hannibal pendant la deuxième guerre punique ${ }^{66}$, avaient en effet été par la suite sévèrement châtiés, perdant plus de la moitié de leur immense territoire ${ }^{67}$. Toutefois, toutes les cités alliées latines et italiennes où TiteLive signale des prodiges pris en charge par Rome ne lui avaient pas été infidèles et n'avaient pas subi de telles confiscations. Loin s'en faut. L'autre exemple invoqué tant par T. Mommsen que par Christopher J. Dart ${ }^{68}$ est celui du prodige in agro publico de Calatia, en 167 av. J.-C. ${ }^{69}$. Or, les éléments rappelés ci-dessus suffisent à prouver qu'elle n'était plus, alors, une cité étrangère. Par conséquent, le prodige in agro publico qui y fut signalé cette année-là ne nous autorise pas à dire : "As such, this example also directly indicates the existence of ager publicus within the territorium of non-Roman communities ${ }^{70} »$.

S'il est légitime d'envisager, à partir de l'exemple lucanien, la possibilité d'expliquer les prodiges signalés dans des cités alliées latines ou italiennes par une localisation sur l'ager publicus, il est en revanche téméraire d'en induire une règle générale en affirmant avec Christopher J. Dart :

- « The portent lists in Livy can in numerous cases be shown to indicate the presence of ager publicus near the reporting community » (p. 112).

- « If portents typically needed to occur over ager publicus in order for them to be considered valid for redress by the Roman state, the portent lists in Livy can be used as an indication of the presence of ager publicus within the territorium of nonRoman communities » (p. 117).

- «As such, the portent lists can be used as a strong indication of the likely presence of ager publicus in Italy » (p. 124)

64 V. Rosenberger, «Prodigien aus Italien: geographische Verteilung und religiöse Kommunikation », CCG 16 (2005), p. 239-240.

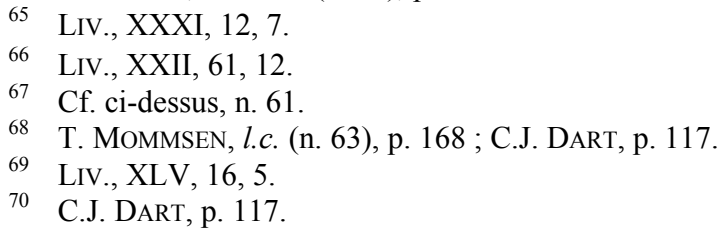


Comme B. MacBain le soulignait déjà dans sa réfutation de l'hypothèse mommsénienne ${ }^{71}$, ce n'est pas parce qu'un prodige, une fois, dans une cité ou une région donnée, est signalé comme s'étant produit in agro publico, que l'on est en droit d'en induire que, dans cette même cité ou région, ainsi que dans les cités ou régions de même statut, les prodiges survinrent à chaque fois sur des terres publiques du peuple romain. L'extrême rareté avec laquelle les listes de prodiges signalent leur localisation in agro publico inviterait plutôt à renverser l'argument : ne serait-ce pas parce qu'il était rare que des prodiges survenus sur l'ager publicus fussent signalés - peut-être parce qu'il était en grande partie constitué de terres de pâturage peu peuplées - qu'on prenait la peine de le mentionner ? Le cas du prodige lucanien in agro publico pourrait être interprété dans ce sens. En effet, dans la même liste de prodiges, quelques lignes seulement avant que soit signalé le prodige survenu sur des terres publiques ${ }^{72}$, un autre prodige est signalé en Lucanie, sans autre précision : in Lucanis caelum arsisse adferebant ${ }^{73}$. Si le signalement de ce dernier prodige avait suivi celui du prodige in agro publico, on aurait pu en induire une localisation identique. Son signalement avant le prodige in agro publico rend à l'inverse cette hypothèse peu probable, même s'il convient, bien sûr, de rester prudent avec les raisonnements a silentio. C'est pourquoi, en l'état actuel de nos connaissances, on ne peut guère s'aventurer au-delà de la démarche de $\mathrm{B}$. MacBain qui, dans l'appendice $B$ de son livre ${ }^{74}$, a pris soin de classer à part, avec les prodiges dont la localisation est trop vague ou d'un statut juridique trop incertain, les prodiges pour lesquels on a de bonnes raisons de soupçonner une localisation sur des terres publiques du peuple romain $^{75}$.

Une localisation in agro publico des prodiges survenus en terre alliée latine ou italienne est d'autant moins plausible que les listes de prodiges nous présentent souvent ces prodiges étrangers comme s'étant déroulés sur les monuments, les portes ou les remparts des cités concernées ${ }^{76}$. Or, il est fort improbable, comme E. Rawson avait raison de le dire ${ }^{77}$, que les monuments, les portes urbaines et les remparts des cités alliées latines ou italiennes aient relevé de la propriété du peuple romain. Tout d'abord, comme on l'a vu, il est difficile de concevoir que des censeurs ou, en leur absence, d'autres magistrats romains, aient pu intervenir sur les murs, les bâtiments et les portes urbaines de ces cités indépendantes. On n'en a, en tout cas, aucune attestation. L'argument des confiscations de terres ne fonctionne pas davantage. Christopher J. Dart écrit, p. 118 : «ager publicus could exist within the territory of Latin and

71 B. MACBAIN, Prodigy and expiation: a study in religion and politics in Republican Rome, Bruxelles, 1982 (Collection Latomus, 177), p. 27.

72 LIV., XXXI, 12, 7.

73 LiV., XXXI, 12, 5.

74 B. MACBAin, o.c. (n. 71), p. 107-113.

75 B. MAcBAIN, o.c. (n. 71), p. 109-110.

76 Ainsi, par exemple, en LIV., XXXII, 1, 10: ab Suessa nuntiatum est duas portas quodque inter eas muri erat de caelo tactum ou en LIV., XXXII, 9, 2 : de caelo tacta erant [...] Herculis aedes Ardeae.

77 E. RAwSON, «Prodigy Lists and the Use of the Annales Maximi», CQ New Series 21.1 (1971), p. 162. Contra C.J. DART, p. 113. 
allied cities. Indeed, all land acquired in war was ager publicus. As Appian sets out, it was traditional practice for newly acquired ager publicus to be leased, sold, or returned to the surrendering community. In numerous cases cities were only returned small portions of their pre-war territorium and left to pay rent on those parts which were now defined as ager publicus ». Comme le rappelle effectivement Appien ${ }^{78}$, et bien d'autres auteurs, à commencer par Tite-Live ${ }^{79}$, les Romains confisquaient souvent ${ }^{80}$ une partie des terres des cités vaincues pour y établir des colonies, les vendre ou les louer comme ager quaestorius ou censorius, ou encore les adjuger à des particuliers sous le régime de la possessio. Toutefois, si l'existence de ces parcelles d'ager publicus confisquées aux cités alliées sans leur être rendues par la suite peut permettre, dans certains cas, comme le cas lucanien, d'expliquer la prise en charge de prodiges signalés sur le territoire - au sens strictement géographique - de telles cités, il ne permet en aucun cas d'expliquer la prise en charge de prodiges survenus sur leurs remparts, leurs portes ou leurs bâtiments urbains. Il est, en effet, absolument invraisemblable que Rome ait confisqué leur territoire urbain aux cités latines ou italiennes qu'elle laissait indépendantes. L'annexion du Picenum l'illustre très clairement : alliés de Rome depuis 299 av. J.-C., les Picentins tentèrent une rébellion en 269, qui aboutit à l'annexion de l'ensemble du Picenum, à l'exception d'une enclave autour d'Asculum - et peut-être autour d'Ancône -, cité à laquelle les Romains décidèrent, précisément, de conserver un statut d'alliée ${ }^{81}$. Lorsque Rome allait jusqu'à confisquer son territoire urbain à une cité vaincue, cela allait de pair avec la suppression de sa souveraineté.

Parmi les cités de droit latin, le cas des colonies latines fondées par Rome est évidemment différent de celui des vieilles cités du Latium restées indépendantes, telles Préneste et Tibur, et l'on pourrait se demander si elles avaient la pleine propriété de leur territoire, de leurs murs et de leurs bâtiments. Mais ce serait oublier que les colonies latines devenaient, une fois fondées ${ }^{82}$, des communautés souveraines sur le plan interne ${ }^{83}$, avec leur propre citoyennete ${ }^{84}$, leurs propres magistrats ${ }^{85}$, leurs

78 ApP., Guerres civiles, I, 7, 26-27.

79 Notamment, Liv., II, 41, 1 ; LIV., X, 1, 3 ; LIV., XXXVI, 39, 3.

80 Le Sénat renonçait parfois à toute confiscation. Cf. le sénatus-consulte de Thisbensibus, 1. 19-20 (IG VII 1, 2225 = FIRA I, 31).

81 Cf. n. 52.

82 Les autorités romaines intervenaient même parfois après la fondation proprement dite, comme le montre l'inscription publiée dans C. ZACCARIA, "Notiziario epigrafico. Aquileia. Novità epigrafiche dal Foro », Aquileia Nostra 67 (1996), col. 179-184, où l'on apprend qu'à l'occasion d'un renforcement de la colonie latine d'Aquilée, décidé en 169 av. J.-C., un des triumvirs fondateurs (en 181), Titus Annius Luscus, érigea un temple, probablement sur le forum, et procéda durant trois années consécutives au recrutement des membres du sénat. Dans la mesure où elle reste étroitement liée à l'acte de fondation, une telle intervention ne peut toutefois pas être interprétée comme une ingérence de Rome dans les affaires internes de la colonie.

83 Voir U. LAFFI, «La colonizzazione romana nell'età della repubblica », in Il Fenomeno coloniale dall'Antichità ad oggi, Accademia nazionale dei Lincei, Giornate dell'Antichità (Roma, 19 e 20 marzo 2002), Roma, 2003 (Atti dei Convegni Lincei, 189), p. 38 [repris dans Colonie e municipi, o.c. (n. 52), p. 16-17]. 
propres assemblées populaires ${ }^{86}$, l'autonomie en matière d'adoption des lois romaines $^{87}$, leur propre juridiction ${ }^{88}$, leur propre cens ${ }^{89}$, leur propre monnaie ${ }^{90}$ et leur propre armée ${ }^{91}$. Elles se distinguaient en cela des colonies de droit romain ${ }^{92}$, qui ne

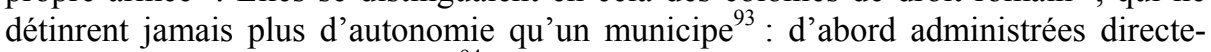
ment par des magistrats romains ${ }^{94}$, elles n'obtinrent leurs propres magistrats que dans un second temps ; encore ceux-ci ne détinrent-ils sans doute d'abord que des compétences limitées ${ }^{95}$, élargies seulement par la suite ${ }^{96}$. Or, la souveraineté d'une communauté s'étend nécessairement sur un territoire. Autrement dit, si une communauté politique était déclarée souveraine par Rome, cette souveraineté s'étendait au territoire de ladite communauté. De fait, la définition de la communauté politique des

84 Cette citoyenneté propre obligeait les citoyens romains qui voulaient s'établir dans une colonie latine à renoncer à la civitas romana.

85 D. KREMER, o.c. (n. 3), p. 67-72.

86 D. KREMER, o.c. (n. 3), p. $72-79$.

87 D. KREMER, o.c. (n. 3), p. 91-95, se refuse à parler de « législation propre », ne serait-ce que parce que le fondateur de la colonie incorporait déjà dans la lex data coloniae des éléments de droit romain.

88 D. Kremer, o.c. (n. 3), p. 95-103. Cf. Cic., Pro Caecina, 98, où il est explicitement dit que les citoyens romains qui partaient s'installer dans une colonie latine le faisaient souvent pour échapper à une peine légale. Voir aussi le cas des triumvirs fondateurs d'Ardée, en 442 av. J.-C., qui échappèrent à d'éventuelles poursuites judiciaires en s'établissant dans la colonie (LiV., IV, 11, 7).

89 D. Kremer, o.c. (n. 3), p. 81-90. En 204 av. J.-C., les douze colonies latines qui s'étaient rebellées cinq ans auparavant (LIV., XXVII, 9, 7) virent leur souveraineté censoriale amoindrie, les autorités romaines leur imposant de procéder désormais au cens selon la formule donnée par les censeurs romains et de venir présenter leurs rôles censoriaux à Rome (LiV., XXIX, 15, 9-10).

90 Voir l'exemple de la monnaie de la colonie latine d'Hadria dans A. CAMPANELLI, « La monetazione di Atri », in Dalla valle del Piomba alla valle del basso Pescara, Fondazione Cassa di Risparmio della Provincia di Teramo, 2001 (Documenti dell'Abruzzo teramano, V.1), p. $94-104$.

91 A.J. ToynBEe, o.c. (n. 3), I, p. 255. Les colonies latines étant dépourvues de toute souveraineté externe, leurs armées étaient au service de la politique étrangère de Rome, à qui elles devaient fournir des contingents.

92 Voir U. LAFFI, « La colonizzazione romana », l.c. (n. 83), p. 38-39 [repris dans Colonie e municipi, o.c. (n. 52), p. 17] ; D. KREMER, o.c. (n. 3), p. 103-106.

93 A.N. SHERwin-White, The Roman Citizenship, Oxford, 1973, p. 86.

94 Comme cela est expressément attesté à Antium : cf. A. DEgRASSI, Scritti vari di antichità, Trieste, 1971, IV, p. 69.

95 E.T. SAlmon, Roman Colonization under the Republic, Ithaca/New York, 1970, p. 80 ; A.N. SHERWIN-White, o.c. (n. 93), p. 77 ; à nuancer avec H. GALSTERER, Herrschaft und Verwaltung im republikanischen Italien. Die Beziehungen Roms zu den italischen Gemeinden vom Latinerfrieden 338 v. Chr. bis zum Bundesgenossenkrieg 91 v. Chr., Munich, 1976, p. 5657.

${ }^{96}$ Peut-être une première fois, à partir de la fin des années 180 av. J.-C., lorsque la taille des colonies de droit romain devint comparable à celles des colonies de droit latin, comme le propose A.N. SHERWIN-WHITE, o.c. (n. 93), p. 84 ; peut-être seulement après la Guerre Sociale, comme le pense A. DEGRASSI, o.c. (n. 94), p. 74. 
colons au moyen de la lustratio effectuée par les deductores romains allait de pair avec la définition, sans doute au moment où ces mêmes deductores énonçaient la lex data coloniae à l'assemblée des nouveaux colons, d'un territoire propre à la colonie ${ }^{97}$. Hyginus nous a gardé un exemple-type de formulation: "en ce qui concerne les terres, les lieux ou les édifices que j'aurai donnés et assignés à l'intérieur de telles limites", par exemple "du cours d'eau un tel, de la voie une telle, que sur ces terres le droit de juridiction et de coercition appartienne à la colonie une telle"98 ». Bien qu'une telle formule de délimitation du territoire colonial ne fût pas spécifique aux colonies latines, il paraît difficile de penser que la portion d'ager publicus populi Romani employée pour y établir une colonie latine pût continuer, par la suite, à relever de la souveraineté romaine. Elle passait plus vraisemblablement, y compris les terres qui n'étaient pas distribuées viritim mais étaient réservées à l'installation des remparts, des portes et des bâtiments publics, sous la souveraineté de la nouvelle colonie $^{99}$. C'est d'ailleurs ce que reconnaît malgré lui Christopher J. Dart qui, tout en considérant que les terres de la colonie qui n'étaient pas distribuées viritim « would thus have remained [Roman] public property and thereby have been subject to taxation » (p. 117), refuse pourtant d'envisager, à la page précédente, que Rome ait pu continuer à exercer sa souveraineté sur le territoire d'une colonie latine : «Fregellae was a Latin colony and so not within the ager Romanus ». Or, comme l'on est bien obligé de considérer que l'ager publicus populi Romani relevait de la souveraineté du peuple romain, il en vient implicitement à limiter l'étendue territoriale de la souveraineté des colons latins aux seuls lots distribués viritim, ce qui revient à nier tout domaine public sur lequel les colonies latines pussent exercer leur souveraineté.

5. Le rejet du prodige de la colonie latine de Frégelles, en 169 av. J.-C. : stricte application d'une règle juridique préexistante ou utilisation pragmatique du droit ?

Abordons, pour terminer, la pièce la plus débattue du dossier, un passage de Tite-Live relatif au refus des autorités romaines, en 169 av. J.-C., de prendre en

97 D.J. Gargola, Lands, Laws, \& Gods: Magistrates \& Ceremony in the Regulation of Public Lands in Republican Rome, Chapel Hill/Londres, 1995, p. 72-82.

98 Hyginus, II, 34 (d'après la numérotation de l'édition de J.-Y. GuILlaumin, Les arpenteurs romains, Paris, 2010, dans la CUF) = HYGINUS, p. 118 Lachmann : 'quos agros, quae loca quaeve aedificia, intra fines' puta 'illos et intra flumen illud, intra viam illam, dedero adsignavero, in eis agris iuris dictio coercitioque esto coloniae illius'. Trad. J.-Y. Guillaumin. Cf. D. J. GARGOLA, o.c. (n. 97), p. 81.

99 Cf. L. CApogrossi-Colognesi, «Le statut des terres dans l'Italie républicaine. Un aspect de la romanisation des campagnes (IV ${ }^{\mathrm{e}}-\mathrm{I}^{\mathrm{er}}$ siècles av. J.-C.) », Histoire et Sociétés Rurales 22.2 (2004), p. 11 : «à cause du caractère formellement étranger des colonies latines à la civitas Romana [...] la division et l'assignation des lots aux colons, en pareil cas, ne soumettent pas ces terres au droit civil romain, mais au droit propre de chaque colonie $»$. 
charge deux des prodiges signalés, dont l'un à Frégelles, colonie latine fondée par Rome en 328 av. J.-C. ${ }^{100}$ :

« Deux prodiges ne furent pas retenus, le premier, parce qu'il s'était produit dans un endroit privé (T. Marcius Figulus annonçait qu'un palmier avait poussé $<$ dans $>$ son impluvium), l'autre, parce que c'était dans un territoire étranger: à Frégelles, dans la maison de L. Atreus, une lance qu'il avait achetée pour son fils, alors soldat, avait, disait-on, brûlé de jour pendant plus de deux heures sans que la moindre parcelle en eût été consumée ${ }^{101} »$.

Rome avait pourtant pris en charge deux précédents prodiges de la colonie latine, en 211 (Anagniae et Fregellis nuntiatum est murum portasque de caelo tacta) et 206 av. J.-C. (nocte Fregellis lucem obortam) ${ }^{102}$. Est-il possible de résoudre une telle contradiction entre la règle énoncée en 169 av. J.-C. et les expiations antérieures de prodiges frégellans, en supposant, sans autre preuve, que le prodige de 169 fut le seul à ne pas s'être produit sur une parcelle d'ager publicus ? C'est extrêmement peu probable : pour les raisons développées plus haut, non seulement le rempart et les portes de Frégelles qui furent frappés par la foudre en 211 av. J.-C. ne pouvaient pas relever de la propriété du peuple romain, mais il ne pouvait de toute façon pas y avoir, à cette date, d'ager publicus populi Romani sur le territoire de Frégelles, colonie latine souveraine et n'ayant subi aucune confiscation foncière de la part de Rome. Reste à réfuter un dernier argument, celui du triomphe refusé, selon Valère Maxime, à Lucius Opimius, qui avait maté la rébellion de 125 av. J.-C. et détruit la colonie latine, quo cautum erat ut pro aucto imperio, non pro reciperatis quae populi Romani fuissent triumphus decerneretur ${ }^{103}$. Selon Christopher J. Dart, p. 117, il faudrait y voir l'indice de la présence d'ager publicus sur le territoire de Frégelles avant 125. Mais il est plus plausible d'interpréter cette décision sénatoriale comme le refus d'accorder un triomphe sur des traîtres, lesquels ne méritent point la dignité d'ennemis du peuple romain : le point commun entre les deux cités évoquées dans l'exemple de Valère Maxime, Capoue et Frégelles, ne porte pas, en effet, sur le statut juridique de leur

100 LiV., VIII, 22, 2 et VIII, 23, 6 ; DenYs D'HaliCARnasse, Antiquités romaines, XV, 8, 5 et XV, 10, 1 (= 15.I. in S. PitTia [éd.], Rome et la conquête de l'Italie aux IV et III ${ }^{e}$ s. av. J.-C., Paris, 2002); cf. APP., Histoire samnite, 4, 1.

${ }_{101}$ LIV., XLIII, 13, 6: Duo non suscepta prodigia sunt, alterum, quod in privato loco factum esset - palmam enatam <in> inpluvio suo T. Marcius Figulus nuntiabat-, alterum, quod in loco peregrino : Fregellis in domo L. Atrei hasta, quam filio militi emerat, interdiu plus duas horas arsisse, ita ut nihil eius ambureret ignis, dicebatur. Trad. P. Jal, CUF, 1976. Cf. D. EngELs, Das römische Vorzeichenwesen (753-27 v. Chr.). Quellen, Terminologie, Kommentar, historische Entwicklung, Stuttgart, 2007 (PAwB, 22), p. 517-521.

${ }^{102}$ En 211 av. J.-C., LIV., XXVI, 23, 5, «à Anagnia et à Frégelles un mur et des portes avaient été frappés par la foudre »; en 206 av. J.-C., LIV., XXVIII, 11, 3, «la nuit, à Frégelles, le jour était apparu ». Trad. P. Jal, CUF, 1991 et 1995. Christopher J. Dart écrit, p. 116 : «Yet Fregellae did report portents which were addressed in 211,206, 197 and then after the rejected portent, again in 169 and $93 \mathrm{BCE} \%$. Le prodige qu'il signale à Frégelles en 197 av. J.-C. s'est plus probablement produit à Fregenae (cf. H. NisSEN, Italische Landeskunde, Berlin, 1902, II.1, p. 350-351 et 351, n. 1), et j'ignore où il a trouvé trace, pour l'année 169 av. J.-C., d'un second prodige frégellan.

103 ValÈre MaXime, Facta et dicta memorabilia II, 8, 4. 
territoire mais sur leur infidélité à Rome. Si l'on suppose que les termes employés par Valère Maxime furent précisément ceux des sénateurs, ce dont on peut évidemment douter, ces derniers, en rappelant que Capoue et Frégelles faisaient déjà partie de l' « imperium romain », c'est-à-dire, dans un sens assez lâche, de la «zone dominée à la fois directement et indirectement par Rome ", ne cherchaient qu'à mieux souligner la trahison de l'un des plus importants municipes romains et de l'une des plus importantes colonies latines fondées par Rome.

C'est pourquoi il convient de chercher ailleurs l'explication du refus des autorités romaines de procurer le prodige frégellan de 169 av. J.-C. Préparant une étude plus approfondie du dossier dans le cadre de mon doctorat, je me contenterai ici de rappeler quelques pistes de réflexion.

Face aux impasses de l'approche strictement juridique de T. Mommsen, B. MacBain a proposé de replacer la prise en charge des prodiges des cités alliées du nomen Latinum et des cités alliées italiennes dans la perspective de leurs relations avec Rome. Ce fut, selon lui, la guerre de Pyrrhus et la guerre d'Hannibal, au cours desquelles la fidélité des alliés fut mise à rude épreuve, qui amenèrent les autorités romaines à prendre en charge des prodiges survenus au-delà des fines populi Romani, afin de réaffirmer symboliquement l'unité politico-religieuse de Rome et de ses alliés ${ }^{104}$. Il mit surtout en avant la capacité d'initiative de ces derniers, soulignant que les prodiges des cités alliées signalés à Rome l'étaient sans doute spontanément, par le biais de leurs autorités ${ }^{105}$. Ce point de vue a le mérite de prendre au sérieux à la fois l'indépendance des cités alliées latines ou italiennes ${ }^{106}$, et l'existence, en contrepoint de l'inclination de Rome à assumer le gouvernement de l'Italie, d'une tendance centripète parmi les alliés ${ }^{107}$. On est dès lors en mesure de comprendre pourquoi Rome prenait en charge des prodiges qui lui étaient spontanément rapportés par ses alliés latins et italiens - elle avait évidemment tout intérêt à ne pas décourager ces signes d'intégration -, et pourquoi Rome fut néanmoins en mesure, en 169 av. J.-C., de refuser cette prise en charge, sous prétexte que le prodige frégellan s'était produit in loco peregrino.

Derrière ce prétexte juridique se cachait en fait probablement, comme B. MacBain l'a supposé ${ }^{108}$, un avertissement diplomatique adressé à la colonie latine. Rome avait en effet, à cette date, plusieurs raisons d'être mécontente de Frégelles. Alors qu'elle était restée fidèle à Rome et s'était même glorieusement illustrée pendant la deuxième guerre punique, Frégelles, qui avait ainsi acquis une importance telle qu'elle devint le porte-parole des colonies latines du centre de la péninsule ${ }^{109}$,

104 B. MAcBAIN, o.c. (n. 71), p. 32.

105 B. MAcBain, o.c. (n. 71), p. 33.

106 Voir, à cet égard, A.N. SHERWIN-White, o.c. (n. 93), p. 127 ; H. MOURITSEN, Italian Unification. A study in ancient and modern historiography, Londres, 1998 (Bulletin of the Institute of classical studies, Suppl. 70), p. 39-40.

107 A.N. Sherwin-White, o.c. (n. 93), p. 128. Cf. J.-M. DAvid, La Romanisation de l'Italie, Paris, 1994, p. 175.

108 B. MAcBain, o.c. (n. 71), p. 28-29.

109 Comme l'illustre clairement le rôle de Lucius Papirius de Frégelles qui s'adressa au Sénat, sans doute en 177 av. J.-C., pro Fregellanis colonisque Latinis : CIC., Brut., 170 ; 
s'était trouvée au cœur des tensions qui naquirent entre Rome et ses alliés latins sur la question du ius migrandi ${ }^{110}$. Ainsi, en 177 av. J.-C., les Samnites et les Péligniens se plaignirent de Frégelles aux autorités romaines car 4000 de leurs familles y avaient émigré, vraisemblablement à la suite de l'émigration massive de Frégellans à Rome $^{111}$. Ces derniers n'étaient d'ailleurs pas les seuls Latins à avoir recouru au ius migrandi dont ils bénéficiaient et à avoir ainsi entraîné vers leur colonie d'origine la migration de populations apennines. Le prouve, par exemple, la présence de Samnites, à cette époque, dans la colonie latine d'Isernia ${ }^{112}$. Or les immigrants latins ne se conformaient pas aux conditions encadrant le ius migrandi. Pour pouvoir en bénéficier, ils devaient laisser dans leur colonie des descendants directs. Or cette stipulation était contournée par bon nombre d'entre eux, qui vendaient leurs fils comme esclaves à des Romains après s'être assurés qu'ils seraient affranchis et recevraient la citoyenneté. Ils en vinrent même, si l'on en croit Tite-Live, à cesser de respecter cette apparence de légalité, en se contentant d'affluer à Rome et de s'y faire recenser ${ }^{113}$. Les autorités romaines finirent par ordonner à deux reprises, en 187 et 177 av. J.-C., l'expulsion des Latins en situation irrégulière et refusèrent, quatre ans plus tard, de recenser ceux qui étaient, malgré tout, restés à Rome ${ }^{114}$. C'est donc là un problème qui paraît avoir empoisonné durablement les relations entre Rome et ses alliés latins, tout particulièrement frégellans. Comme F. Coarelli l'a rappelé, ces épisodes de migration massive s'expliquent par les profondes mutations socio-économiques que connut alors une grande partie de l'Italie, qui s'accompagnèrent d'un enrichissement des classes dirigeantes romaines, latines et, plus généralement, italiennes, et d'un appauvrissement des classes inférieures. Il en résulta des tensions d'une grande complexité qui convergèrent, pour des motifs différents d'une classe sociale à l'autre, sur la question de l'accès des alliés latins et italiens à la citoyenneté romaine. Telle fut la matrice de la révolte de Frégelles, en 125 av. J.-C., après le rejet de la proposition

cf. LiV., XLI, 8, 6-12. Voir F. CoArelli, « La storia e lo scavo », in F. CoARELli, P. G. Monti (éds), Fregellae, Rome, 1998, I. « Le fonti, la storia, il territorio », p. 34-35.

110 W. BROADHEAD, «Rome's migration policy and the so-called ius migrandi », CCG 12 (2001), p. 69-89, a contesté l'existence du ius migrandi. Ce prétendu « droit », loin d'avoir été un privilège accordé aux Latins, aurait été une exceptio restreignant le droit de migration des colons latins afin de s'assurer que les effectifs militaires des colonies ne seraient pas affaiblis par des émigrations massives vers Rome. Si son interprétation s'avérait juste, on comprendrait mieux encore les raisons du mécontentement des autorités romaines et de l'avertissement qu'elles adressèrent aux Frégellans en 169 av. J.-C.

111 Liv., XLI, 8, 8. Cf. F. CoARELLI, «I Sanniti a Fregellae», dans La Romanisation $d u$ Samnium aux II et $I^{e r}$ siècles av. J.-C., Actes du colloque organisé par le Centre Jean Bérard (Naples, 4-5 novembre 1988), Naples, 1991 (Collection du Centre Jean Bérard, série 2, vol. 9), p. $177-185$.

${ }^{112}$ CIL I' ${ }^{2}$, 3201 ; A. LA Regina, «I territori sabellici e sannitici », DArch 4-5 (19701971), p. 452-453; id., «I Sanniti », in C. Ampolo, D. Briquel, P. CÀssola Guida et al. (éds), Italia, omnium terrarum parens. La civiltà degli Enotri, Choni, Ausoni, Sanniti, Lucani, Bretti, Sicani, Siculi, Elimi, Milan, 1989, p. 308-309.

${ }_{113}$ LIV., XLI, 8, 9-11.

114 Liv., XXXIX, 3, 4-6 (187 av. J.-C.) ; LIV., XLI, 8, 12 et XLI, 9, 9-12 (177 av. J.-C.) ; LIV., XLII, 10, 3 (173 av. J.-C.). 
de loi du consul Marcus Fulvius Flaccus concédant la citoyenneté à l'ensemble des Italiens, révolte qui aboutit à la destruction de la colonie latine ${ }^{115}$.

L'autre élément contextuel du refus du prodige frégellan, en 169 av. J.-C., réside dans la guerre que les Romains livraient au roi de Macédoine, Persée. L'hasta destinée au fils de Lucius Atreus qui brûla, dit-on, pendant plus de deux heures sans se consumer était une arme typique de la cavalerie, ce qui rend probable l'identification de son propriétaire comme un soldat de la turma Fregellana, dont le rôle lors de la guerre est attesté par la mission que dut remplir chez les Pénestes et les Parthiniens un certain Marcus Trebellius de Frégelles ${ }^{116}$. L'autre prodige refusé par les autorités romaines peut lui aussi être mis en rapport avec la guerre de Macédoine : le palmier, signe de victoire, qui, selon Titus Marcius Figulus, avait poussé dans son impluvium, doit être compris comme le signe de la prétention des Marcii à obtenir des commandements, âprement disputés, dans la guerre contre Persée : ce fut d'ailleurs le consul Quintus Marcius Philippus qui obtint la charge de la Macédoine et le préteur Gaius Marcius Figulus le commandement de la flotte ${ }^{117}$.

Mutations et tensions socio-économiques manifestées par des phénomènes de migration massive de Latins vers Rome, souvent au mépris du droit, et d'Italiens vers les colonies latines, compétition aristocratique aussi bien au sein des classes dirigeantes latines que romaines, telle est la toile de fond, malheureusement très incomplète, sur laquelle s'inscrit le rejet du prodige frégellan de 169 av. J.-C. Si ce double contexte ne nous permet pas de comprendre jusqu'au bout les motivations du refus des autorités romaines, il nous incite à ne pas y voir la simple application d'une règle juridique. Les termes mêmes de la source, probablement sénatoriale, que suit ici Tite-Live ${ }^{118}$, invitent plutôt à l'interpréter comme un message diplomatique appuyé sur un prétexte juridique ${ }^{119}$. Il est dit, en effet, que le prodige fut rejeté non pas parce qu'il s'était produit in agro peregrino mais in loco peregrino: par son emploi en dehors de l'expression augurale figée ager peregrinus, l'adjectif peregrinus voit son sens plein d' " étranger» réactivé, comme si les autorités romaines avaient voulu rappeler aux Frégellans que leur colonie, en dépit des liens étroits qui l'unissaient à Rome, restait une simple cité « étrangère ${ }^{120}$. Le message s'adressait-il aux autorités de Frégelles? aux Frégellans qui continuaient à abuser du ius migrandi ? au seul Lucius Atreus ? Cela est difficile à dire, même si la dernière hypothèse est affaiblie par le rejet du prodige au motif d'une localisation in loco peregrino et non pas,

115 F. COARELLI, l.c. (n. 109), p. 41.

116 Liv., XLIII, 13, 6 et XLIII, 21, 2-3. Cf. E. RAwson, « Fregellae: Fall and Survival », in F. CoArelli, P. G. Monti (éds), o.c. (n. 109), p. 71 et, ibidem, F. CoArelli, l.c. (n. 109), p. 34

117 LiV., XLIII, 15, 3. Cf. F. Münzer, s.v. Marcius 65), RE XIV.2 (1930), col. 1560 ; B. MACBAin, o.c. (n. 71), p. 30.

118 S.W. Rasmussen, Public Portents in Republican Rome, Rome, 2003, p. 220 ; D. ENGELS, o.c. (n. 101), p. 518.

119 Dans la mesure où les autorités romaines acceptaient de prendre en charge d'autres prodiges survenus in loco privato, en particulier les naissances de monstres humains, le motif avancé pour le rejet du prodige de Titus Marcius Figulus semble également avoir été un prétexte juridique : B. MACBAIN, o.c. (n. 71), p. 30.

120 Cf. P. CATALANO, l.c. (n. 9), p. 496-497. 
comme dans le cas du palmier de Titus Marcius Figulus, in loco privato. Quoi qu'il en soit, l'avertissement ne semble pas avoir été bien sévère : diplomatiquement présenté comme la stricte application d'une norme juridique, le rejet des prodiges de Lucius Atreus et de Titus Marcius Figulus n'empêcha ni les aristocrates frégellans ni les Marcii, on l'a vu, de s'illustrer dans la guerre de Macédoine.

Attaché Temporaire d'Enseignement et de Recherche,

Yann BERTHELET

Université Paris 1 Panthéon-Sorbonne

Doctorant rattaché à l'UMR 8210 ANHIMA

(Anthropologie et Histoire des Mondes Antiques)

Rue Vivienne, 2

F-75002 Paris

yann.berthelet@normalesup.org 